\title{
Pulse propagation in discrete excitatory networks of integrate-and-fire neurons
}

\author{
Laurent Badel* ${ }^{*}$ and Arnaud Tonnelier ${ }^{\dagger}$ \\ Laboratory of Computational Neuroscience, Swiss Federal Institute of Technology, Lausanne, Switzerland
}

(Received 16 May 2003; revised manuscript received 20 February 2004; published 12 July 2004)

\begin{abstract}
We study the propagation of solitary waves in a discrete excitatory network of integrate-and-fire neurons. We show the existence and the stability of a fast wave and a family of slow waves. Fast waves are similar to those already described in continuum networks. Stable slow waves have not been previously reported in purely excitatory networks and their propagation is particular to the discrete nature of the network. The robustness of our results is studied in the presence of noise.
\end{abstract}

DOI: 10.1103/PhysRevE.70.011906

PACS number(s): 87.10.+e, 87.19.La, 05.45.Ra

\section{INTRODUCTION}

Neurons in the brain interact by short electrical pulses. Homogeneous networks of pulse emitting units such as neurons can be in a state of synchronous [1] or asynchronous activity $[2,3]$. In networks with spatial topology, additional states are possible that are characterized by the propagation of wavelike activity patterns [4-9]. Recently, a number of experiments have indicated the existence of propagating activity waves in neuronal tissue, notably the cortex [10,11], thalamus [12-14], and hippocampus [15]. The mechanism underlying the wave propagation is believed to be synaptic in origin and has recently been the subject of several theoretical studies [4-9]. Most of the theory has been developed in the framework of continuum models that approximate networks made of discrete elements. However it is well known that discrete systems can exhibit behaviors that are not present in their continuous counterparts. The most eloquent example is the occurrence of propagation failure in discrete diffusive media [16].

In this paper we explore the effects of the discrete spatial structure on pulse propagation in a network of excitable cells. We focus on excitatory networks which have retained the widest attention in earlier studies [4-7]. Detailed models are difficult to analyze and we consider a simplified description for the kinetics of single cells that retains the phenomenological dynamics of spiking neurons [17]. Using a network of integrate-and-fire neurons, we exactly determine the velocity and stability of propagating pulses. We find a fast wave and a family of slow waves. The fast wave is similar to the fast pulse already reported in continuum models while slow waves are characterized by a nonmonotonic approach towards the threshold. The existence of the fast wave is robust in the sense that a strong coupling strength is sufficient to guarantee its existence whereas the family of slow waves depends strongly on the form of the coupling and the length of the synaptic footprint. Furthermore, we study the effects of noise on pulse propagation and we observe a transition

\footnotetext{
*Corresponding author. Electronic address: laurent@badel.org

${ }^{\dagger}$ Current address: CORTEX project, INRIA Lorraine, Campus Scientifique, 54602 Villers-lès-Nancy, France. Electronic address: arnaud.tonnelier@loria.fr
}

between different stable waves, most often toward faster solutions. In previous studies $[9,18]$ stable slow waves have been found for interacting populations of excitatory and inhibitory neurons. Here we report slow waves that arise due to the discrete topology of the network. In the continuum limit, i.e., in the limit of a large number of units and connections, the slow waves disappear and we recover results already obtained in spatially continuous excitatory networks.

\section{THE MODEL}

We consider an infinite one-dimensional network of excitatory neurons where each neuron is allowed to fire only one spike. A neuron located at $i \in \mathbb{Z}$ is described, at time $t$, by its membrane potential $v_{i}(t)$. The spiking process is described by the firing time $t_{i}^{(f)}$ defined by the moment of threshold crossing, $v_{i}\left(t_{i}^{(f)}\right)=\vartheta$, from below, $d v_{i}(t) /\left.d t\right|_{t=t_{i}^{(f)}}>0$. The subthreshold dynamics is given by the leaky integrate-and-fire model,

$$
\frac{d v_{i}(t)}{d t}=-\frac{v_{i}(t)}{\tau}+I_{i}(t)+\left(v_{r}-\vartheta\right) \delta\left(t-t_{i}^{(f)}\right)
$$

where $\tau$ is the membrane time constant, $I_{i}$ denotes the total input current for neuron $i$ (normalized by the membrane capacitance), and $\delta$ is the Dirac pulse. The last term on the right-hand side of Eq. (1) is a reset current that leads to the reset process: when $v_{i}$ reaches the firing threshold $\vartheta$, it is immediately reset to the subthreshold value $v_{r}$.

Each neuron receives inputs from its $N$ nearest neighbors. The input current is taken to be of the form

$$
I_{i}(t)=g_{s y n} \sum_{|i-j| \leqslant N} \omega_{i j} \alpha\left(t-t_{j}^{(f)}\right)+I_{\mathrm{a} p p},
$$

where $I_{\mathrm{a} p p}$ is an applied current which we set to zero since we are interested in the excitable regime only, $g_{s y n}$ is a positive coupling parameter, $N$ is the distance of interaction, and $\alpha$ is some type of $\alpha$-function describing the effect of a single incoming spike. Its precise shape is crucial for the existence of slow waves, as will be discussed later. For a better control on the time constants of $\alpha$ we use the piecewise linear representation 


$$
\alpha(t)=\left\{\begin{array}{cc}
t / \tau_{r}, & 0 \leqslant t \leqslant \tau_{r} \\
1+\left(\tau_{r}-t\right) / \tau_{d}, & \tau_{r} \leqslant t \leqslant \tau_{r}+\tau_{d} \\
0, & \text { otherwise }
\end{array}\right.
$$

where $\tau_{r}$ is the synaptic rise time and $\tau_{d}$ is the synaptic decay time. However, our main results still hold with smoother functions. The synaptic weights $\omega_{i j}$ are functions of the distance between neurons $i$ and $j$ only, that is, $\omega_{i j}=\omega(|i-j|)$, where $\omega(r)$ is the synaptic footprint. The qualitative results do not depend on the precise form of $\omega$ and standard forms or a random function can be considered. For concreteness, unless stated otherwise, numerical simulations are done using the square footprint shape: $\omega(r)=(2 \sigma)^{-1}$ for $|r|<\sigma$ and 0 otherwise.

\section{TRAVELING WAVE SOLUTIONS}

We look for traveling waves where each neuron fires exactly one spike. Integration of Eq. (1) over the interval $(-\infty, t]$ yields the following expression for $v_{i}$ :

$$
v_{i}(t)=\eta\left(t-t_{i}^{(f)}\right)+g_{s y n} \sum_{j=-N}^{N} \omega_{j} \epsilon\left(t-t_{i-j}^{(f)}\right),
$$

where $\eta(t)=\left(v_{r}-\vartheta\right) e^{-t / \tau} \Theta(t)$, with $\Theta$ the Heaviside step function, results from the reset process and $\epsilon(t)$ $=\int_{0}^{t} \alpha(s) e^{-(t-s) / \tau} d s \Theta(t)$ is the normalized EPSP (excitatory postsynaptic potential). We have written $\omega_{j}$ instead of $\omega_{i, i-j}$ so as to simplify the notations; we shall do so throughout this paper.

We define a traveling wave solution of Eq. (1) to be one for which $v_{i}(t)=V(t-i / c)$, where $c>0$ is the velocity. The requirement that each neuron fires only once implies that $t_{i}^{(f)}=i / c$ (up to an arbitrary constant due to the translation invariance of the traveling wave solution) and subsequently that $V(\xi=0)=\vartheta$, where $\xi=t-i / c$ is the traveling wave coordinate. Substitution in Eq. (3) yields

$$
V(\xi)=\eta(\xi)+g_{\text {syn }} \sum_{j=1}^{N} \omega_{j} \epsilon(\xi+j / c) .
$$

The sum runs over positive $j$ only since $\epsilon(\xi)$ vanishes for $\xi$ $\leqslant 0$. Using $V(0)=\vartheta$, we obtain the self-consistency condition for the wave speed $c$,

$$
\sum_{j=1}^{N} \omega_{j} \epsilon(j / c)=\frac{\vartheta}{g_{s y n}} .
$$

This equation accompanied by the condition

$$
V(\xi)<\vartheta \quad \text { for } \xi<0
$$

which states that neurons reach the threshold for the first time at $\xi=0$, determines the existence and the speed of traveling waves in the network. The corresponding wave profile is given by Eq. (4).

As it is commonly done for the study of continuum networks [5,8], the stability of traveling waves is calculated by considering perturbations of the firing times. This technique
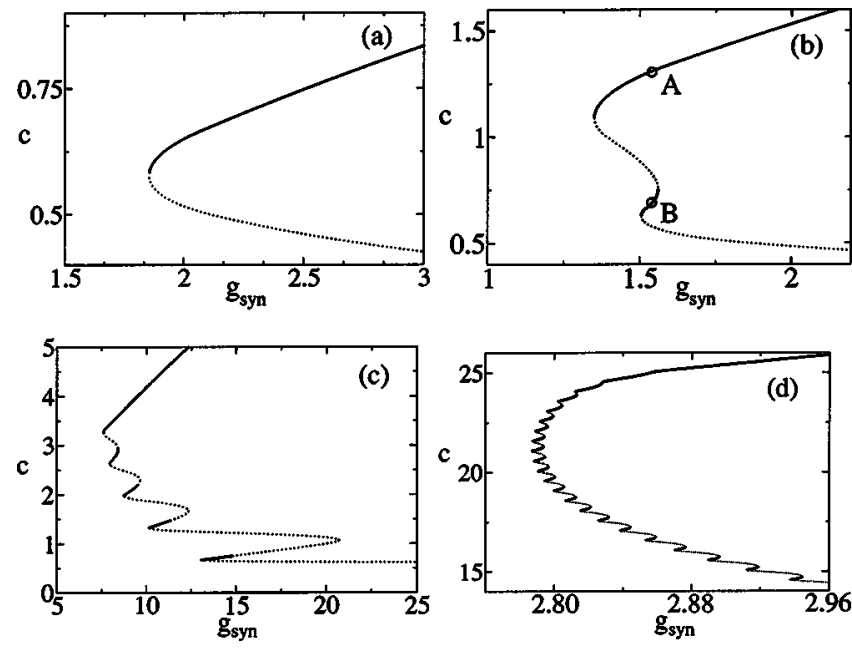

FIG. 1. The speed $c$ of a traveling pulse as a function of the coupling parameter $g_{s y n}$ is shown for various numbers $N$ of presynaptic neurons. Figures are obtained by solving Eq. (5) numerically. Solid lines indicate stable solutions and dotted lines denote solutions that are unstable or unacceptable [with respect to criterion (6)]. Parameters are $\vartheta=1, \tau=1, \tau_{r}=1.5$ and (a) $N=1, \tau_{d}=0.5$, (b) $N=2, \tau_{d}=0.5$, (c) $N=5, \tau=0.1, \tau_{d}=0.1$, and (d) $N=50, \tau=0.05, \tau_{r}$ $=1.99, \tau_{d}=0.01$. Calculations were done in (c) and (d) using the exponential footprint $\omega_{i}=\exp (-i / 5)$ and $\omega_{i}=\exp (-i / 25)$, respectively (for the square footprint, the corresponding figure shows tiny stable branches of slow pulse that are difficult to visualize). The normalization is achieved by rescaling $g_{s y n}$. When $N \geqslant 2$ there is a family of slow waves. The circles labeled A and B in (b) at $g_{s y n}$ $=1.54$ denote a reference parameter set related to the fast and the slow wave, respectively.

restricts the analysis to perturbations that travel with the same velocity as the wave. If we suppose that $t_{j}^{(f)}=j / c+u_{j}$, where $u_{j}$ is a small perturbation, asymptotic stability holds if $u_{j} \rightarrow 0$ as $j \rightarrow \infty$. Expanding Eq. (3) to first order in $u_{j}$ and assuming a perturbation of the form $u_{j}=e^{\lambda j}$ yields the characteristic equation

$$
\sum_{j=1}^{N} \omega_{j}\left(e^{-\lambda j}-1\right) \epsilon^{\prime}(j / c)=0
$$

where the derivative $\epsilon^{\prime}$ can be expressed as the difference,

$$
\epsilon^{\prime}(x)=\alpha(x)-\frac{1}{\tau} \epsilon(x) .
$$

Asymptotic stability holds if all nonzero solutions of Eq. (7) have a negative real part. Note that $\lambda=0$ is always a solution due to translation invariance. Numerically, we find that branches along which $d g_{s y n} / d c>0$ are stable, all others being unstable. A pulse wave can lose its stability through a saddle-node bifurcation. Other bifurcations do not appear in our analysis.

Using Eq. (5) together with results of the stability analysis, we plot in Fig. 1 the wave speed as a function of the coupling parameter $g_{s y n}$. For nearest-neighbor couplings, i.e., 


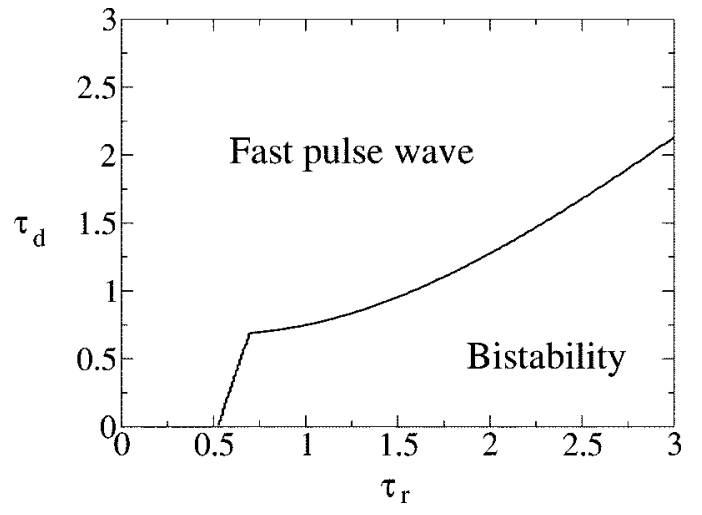

FIG. 2. Locus of existence of a bistable regime, i.e., the existence and the stability of a fast pulse and a slow pulse, in the $\left(\tau_{r}, \tau_{d}\right)$ plane. Parameters are $\vartheta=1, \tau=1, N=2$. In the bistable region (lower part of the graph) there exist values of the coupling strength $g_{\text {syn }}$ such that a fast wave and a slow wave can propagate in the network. In the upper part, only fast waves can be initiated.

$N=1$, we qualitatively obtain the same results as those obtained in continuum models. Specifically, we obtain the following.

(i) There is a critical coupling $g_{\text {syn }}^{*}$ below which the wave fails to propagate.

(ii) There are two branches of solutions for the wave speed reported as the fast and the slow branch.

(iii) The solutions on the slow branch are irrelevant since they are unstable or do not satisfy the condition (6).

As the extent of the coupling increases, that is, when $N$ $\geqslant 2$, a qualitative change occurs in the form of the speed graph as new branches of stable solutions appear. The number of these branches is parametrized by the number $N$ of presynaptic neurons, and their existence depends crucially on the form of $\alpha$. As we have already mentioned, the shape of the weight function does not qualitatively affect this result but changes the size of the regions of stability. The exact conditions under which the slow waves begin to exist are difficult to establish. A necessary, but not sufficient condition is $\alpha$ having a sharp decaying phase. Let us focus on the case
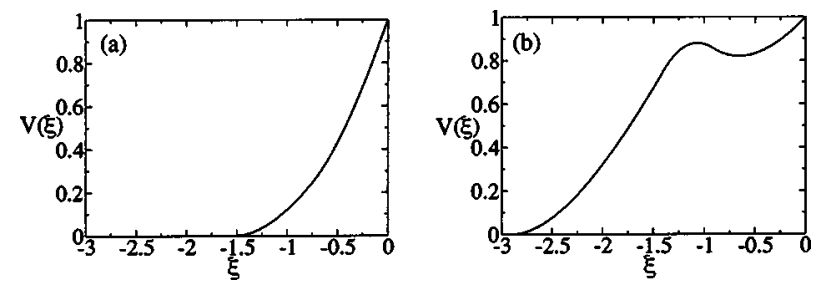

FIG. 3. The wave profile $V(\xi)$ as a function of the traveling wave coordinate $\xi$ for $\xi<0$ (neurons have not fired) obtained by a numerical simulation of the network. Results are in agreement with the theoretical expression (4). The membrane potential of (a) the fast wave and (b) the slow wave are shown, corresponding to the two points A and B of Fig. 1(b), respectively.

$N=2$ which exemplifies much of the interesting features. In this case, there is at most one stable branch of slow waves. The bifurcation diagram shown in Fig. 2 gives the precise location of the slow waves in the two-parameter diagram $\left(\tau_{r}, \tau_{d}\right)$. It can be seen that a simple necessary condition for the existence of the bistable regime is given by $\tau_{r}>\tau_{d}$. The typical profile of the two waves is shown in Fig. 3 for the two solutions marked as A and B in Fig. 1(b). The potential of the fast wave increases monotonically to the threshold while the slow wave profile has a bump. This is due to the fact that the firing period $1 / c$ is comparable to the time to peak of $\epsilon$. This results in the effect of the first spike received from the second neighbor beginning to wear off shortly after the arrival of the second spike. The selection between the two waves is made by the initial stimulation. The fast wave is easily initiated with a shock initial condition while the slow wave is evoked when forcing the neighboring neurons to fire one after another with a delay close to $1 / c$. In practice the first neurons of the network are stimulated using the theoretical prediction of the wave profile given by Eq. (4). The two types of pulse are observed from simulating Eq. (1) with different initial stimulations (Fig. 4).

These observations generalize to the case of $N$ presynaptic neurons: up to $N-1$ branches of stable slow waves can be expected. On the fast solution branch, the corresponding voltage increases monotonically before hitting the threshold,
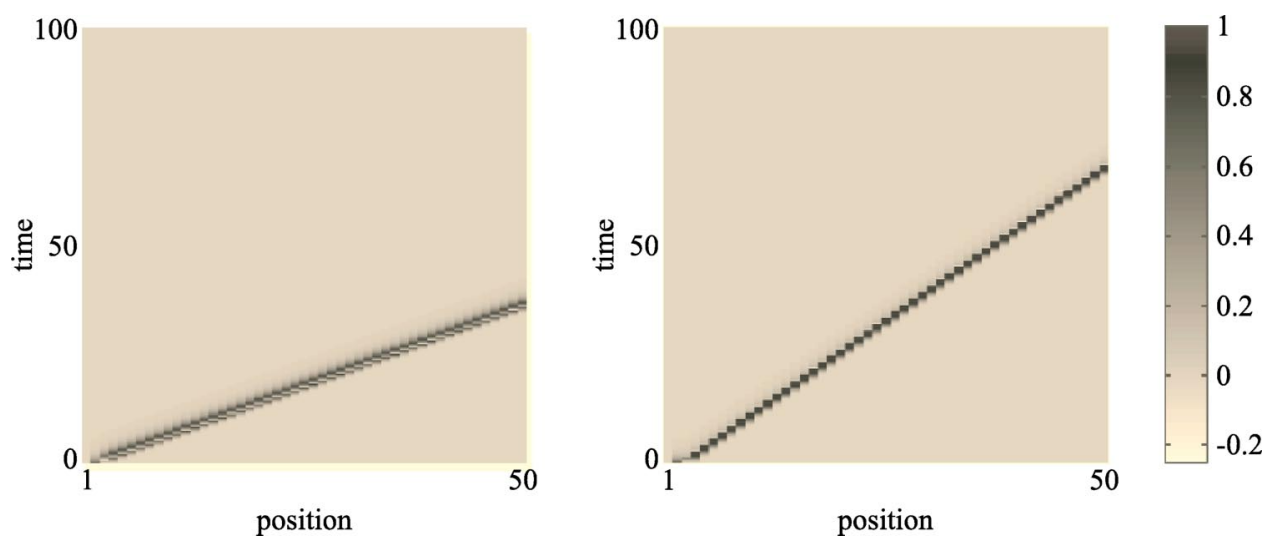

FIG. 4. Space-time plot of voltage obtained by a numerical integration of Eq. (1) in a network of 50 neurons using the reference parameter set given in Fig. 1. The voltage is shown in levels of gray according to the scale bar on the right. The condition that each neuron can fire only one spike is fulfilled by taking a sufficiently small reset value, $v_{r}=-0.25$. The two types of pulses are initiated using an injected current into a group of $N$ neurons on the left. Here $N=2$. 
whereas on the slow branches it has a number of maxima (in general, with decreasing wave speed each new branch of solutions is characterized by an additional maximum, thus there can be up to $N-1$ ). It can be seen from Fig. 1(d) that the branches of slow solutions may overlap, resulting in a multistable regime. As $N$ is increased, the slow solutions progressively disappear, as the size of the corresponding branches diminishes; the number of folds in the speed graph increases according to $N$ and the folds become thinner at the same time. In the continuum limit, obtained as $N \rightarrow \infty$ while the distance between cells is rescaled as $1 / N$, the speed diagram approaches that of the corresponding continuum model in which no slow wave exists [Fig. 1(d)]. Note that for sufficiently strong coupling, there is only a fast wave since the slow one is unstable (or unacceptable) and the wave speed is mainly determined by the firing of the most remote presynaptic neuron, although contributions of other presynaptic neurons tend to increase the wave speed.

\section{SOME ANALYTICAL RESULTS}

We now determine some properties of traveling waves by using the specific shape of $\alpha$ or by taking advantage of some limiting situations. From Eq. (2) we calculate the analytical expression of the EPSP, $\epsilon$. The self-consistency equation (5) can be rewritten as

$$
\gamma_{N+1} z+\sum_{j=0}^{N} \gamma_{i} e^{-j z}=\frac{\vartheta}{g_{s y n}},
$$

where $z=1 / c$ and the constants $\gamma_{i}$ depend on the parameters of the network: the time constants $\tau, \tau_{r}, \tau_{d}$ and the synaptic weights $\omega_{i}$. The wave speed can be explicitly calculated in the simple case of nearest-neighbor connections, i.e., $N=1$, and we obtain (Appendix A)

$$
c=\left\{\begin{array}{lc}
\frac{1}{\tau\left[W_{-1}\left(-\gamma e^{-a}\right)+a\right]}, & c^{*} \leqslant c \leqslant \tau_{r}^{-1} \\
\frac{1}{\tau\left[W_{0}\left(-e^{-b}\right)+b\right]}, & c>\tau_{r}^{-1},
\end{array}\right.
$$

where $W_{k}$ denotes the $k$ th branch of the Lambert $W$ function [19] (the expression of the constants $a, b$, and $\gamma$ is given in the Appendix). The speed $c^{*}$ denotes the minimal wave speed given by

$$
c^{*}=\left\{\tau_{r}+\tau \ln \left[1+\left(\tau_{d} / \tau_{r}\right)\left(1-e^{-\tau_{r} / \tau}\right)\right]\right\}^{-1}
$$

and obtained from the critical coupling

$$
g_{\text {syn }}^{*}=\vartheta /\left\{\tau-\left(\tau^{2} / \tau_{d}\right) \ln \left[1+\left(\tau_{d} / \tau_{r}\right)\left(1-e^{-\tau_{r} / \tau}\right)\right]\right\}
$$

below which propagation fails. Note that we have incorporated the coupling strength $\omega_{1}$ in $g_{s y n}^{*}$, i.e., we take $\omega_{1}=1$. Equation (11) gives an upper bound for $g_{s y n}^{*}$ as $N$ varies (when introducing the normalization of the synaptic weights $\omega_{j}$ in $g_{s y n}$ ). Moreover, it is straightforward to derive a general scaling law for the velocity as a function of the coupling strength (see Appendix B)

$$
c^{q+1}=K g_{s y n},
$$

as $c \rightarrow \infty$, where $q$ is the order of the first nonzero term in the expansion of $\alpha(t)$ at $t=0$, and $K$ is a constant. For $\alpha$ given by Eq. (2), we have $q=1$.

\section{EFFECTS OF NOISE}

Real neurons are subject to noise which causes their physical properties to fluctuate. There are numerous possibilities for modeling noise in spiking neurons and we consider here the simple case where the firing threshold is the noisy parameter. In principle, any of the neuronal parameters can be subject to this type of noise but for the sake of simplicity we will concentrate on a "slow noise in the threshold" [17]. Due to the linearity of the model, this approach is formally equivalent to a fluctuating conductance $g_{s y n}$. In the following, the firing thresholds of the neurons are treated as independent, identically distributed random variables with a given distribution $P_{\vartheta}$ which we assume to be Gaussian, i.e.,

$$
P_{\vartheta}(x)=\frac{e^{-(x-\tilde{\vartheta})^{2} / 2 \sigma}}{\sqrt{2 \pi \sigma^{2}}}
$$

where $\widetilde{\vartheta}=1$ is the center of the distribution, and $\sigma \ll 1$ its width. In this situation, it is no longer possible to predict the firing times given the input, but only their probability distribution. In the noiseless case, i.e., $\sigma=0$, the network has up to $N$ stable waves and we can expect that for sufficiently small amounts of noise the network fluctuates around these solutions. The perturbation of the firing time of neurons will propagate along the network with the wave of excitation. If we can show that there is a high probability that this perturbation stays bounded, then the wave is "statistically" stable. To clearly illustrate our analysis, we focus on the case $N$ $=2$, but the same technique can be applied to any $N$.

Let $T=c^{-1}$ be the time (in the noiseless network) between the spikes of two neighboring neurons (say, $i=0$ and $i=1$ ). Given two presynaptic firing times $t_{0}^{f}=0$ and $t_{1}^{f}=T-\delta t_{1}$, where $\delta t_{1}$ is a perturbation due to noise, the membrane potential of the postsynaptic neuron evolves according to Eq. (3),

$$
v\left(t \mid \delta t_{1}\right)=g_{s y n}\left[\omega_{2} \epsilon(t)+\omega_{1} \epsilon\left(t-T+\delta t_{1}\right)\right],
$$

and firing occurs at $t_{2}^{f}=\left(T-\delta t_{1}\right)+\left(T-\delta t_{2}\right)$ if

$$
v\left(2 T-\delta t_{2}-\delta t_{1} \mid \delta t_{1}\right)=\vartheta,
$$

with $\vartheta$ the actual value of the threshold, provided the threshold has not been reached at an earlier time (for convenience, we will write $s=\delta t_{1}$ and $r=\delta t_{2}$ ). Hence, the probability of occurrence of $r$ is zero if this condition is violated, and is otherwise determined by the threshold distribution through the relation

$$
P(r \mid s)=P_{\vartheta}(v(2 T-r-s \mid s))\left|\frac{d v}{d r}\right| .
$$

In addition, the neuron fails to fire if $\max \{v(t \mid s)\}<\vartheta$, which leads to the normalization 


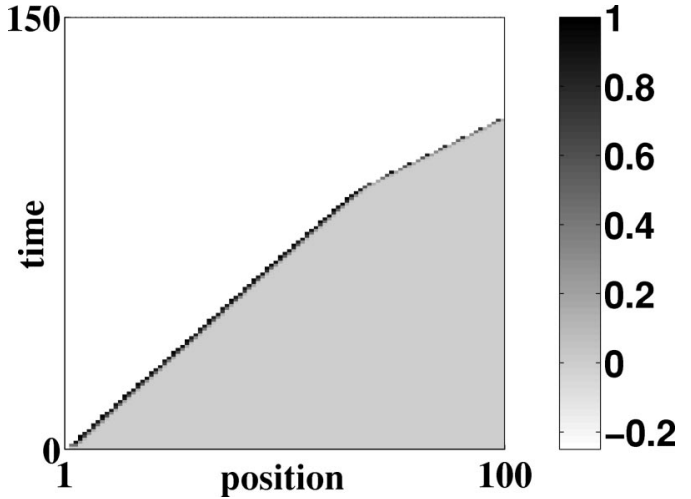

FIG. 5. Transition from a slow wave to a fast wave in a noisy network, obtained by numerical integration of Eq. (1) in a network of 100 neurons with noisy thresholds. The voltage is shown in levels of gray according to the scale bar on the right. Parameters are $\tau=1, \tau_{r}=1.5, \tau_{d}=0.5, g_{\text {syn }}=1.56$, and $\sigma=0.01$ (see Fig. 6 for a comparison). The slow wave is initiated by stimulating a group of $N$ neurons on the left (here, $N=2$ ). All neurons are held silent after spiking for the first time.

$$
P^{f a i l}(s)+\int_{-\infty}^{\infty} d r P(r \mid s)=1,
$$

where

$$
P^{f a i l}(s)=\int_{\max \{v(t \mid s)\}}^{\infty} d x P_{\vartheta}(x)
$$

is the failure probability. Calculating $P(r \mid s)$ and $P^{f a i l}(s)$, we obtain the following results: for small $|s|$ and $\sigma$, the distribution of $r$ is peaked around $r=0$ and the failure probability is negligible, i.e., the network tends to fluctuate around the noiseless pulse wave solution. For large values of $|s|$ or $\sigma$, $P^{\text {fail }}$ approaches 1 which leads to a high probability of propagation failure. For the slow wave, there is also an intermediate domain, when $s$ is positive but not too large, where the failure probability is low, but another peak emerges about $r$ $=s$ in the firing-time distribution; the network has a high probability of switching to the fast wave solution (Fig. 5). These observations are corroborated by the iterative calculation of the firing-time distribution

$$
P_{n}(r)=\int d s P(r \mid s) P_{n-1}(s)
$$

with the initial condition

$$
P_{0}(r)=\delta(r)
$$

that is, the noise is "switched on" when the wave reaches the neuron located at $i=1$. The evolution of the firing-time distribution (see Fig. 6) clearly shows three possible outcomes.

(i) When $\sigma$ is small enough, the slow wave is "statistically" stable but because of the noisy threshold, the failure probability always has a small positive value.

(ii) As $\sigma$ grows, the slow wave destabilizes before the fast wave. The robustness of the fast wave could be guessed from Eq. (13) since $d v / d r$ is significantly larger for the fast
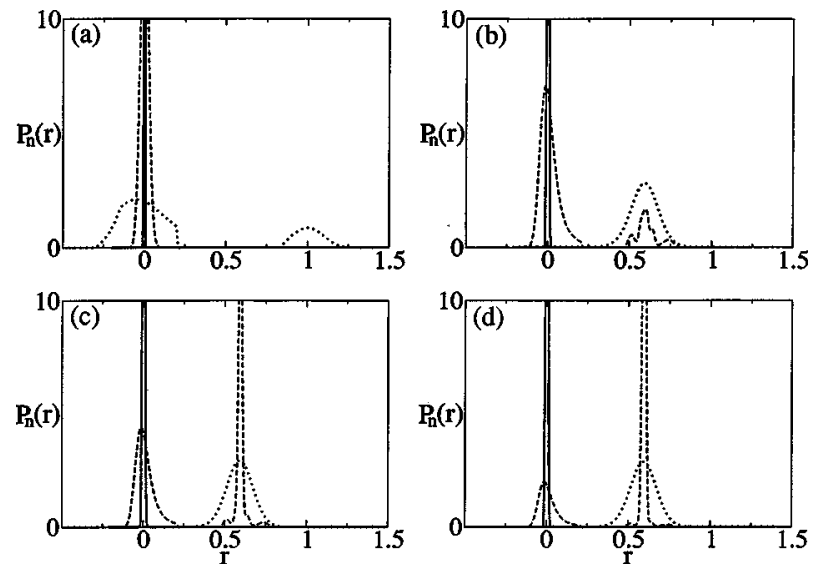

FIG. 6. Firing-time distributions $P_{n}(r)$ associated to the slow wave solution for various amounts of noise: $\sigma=0.001$ (solid line), $\sigma=0.01$ (dashed line), and $\sigma=0.08$ (dotted line). The evolution of the firing-time distribution is represented at (a) $n=1$, (b) $n=11$, (c) $n=26$, (d) $n=51$. Parameters of the network are $\tau=1, \tau_{r}=1.5, \tau_{d}$ $=0.5, g_{\text {syn }}=1.56$. The speed of the slow and the fast wave is $c_{\text {slow }}$ $=0.74$ and $c_{\text {fast }}=1.32$, respectively. The peak on the right-hand side is centered around $0.6 \simeq 1 / c_{\text {slow }}-1 / c_{\text {fast }}$ and corresponds to the probability that the network switches to the fast wave solution.

wave, so that a given perturbation on the threshold results in a smaller perturbation of the firing time. Thus, there is a regime where only fast waves can propagate onto significant distances.

(iii) For large values of $\sigma$ both waves destabilize and the network returns to its resting state. Note that the width of the distribution $P_{n}(r)$ also provides an estimate of the fluctuations in the interspike intervals.

\section{HIGHER-DIMENSIONAL NETWORKS}

We focus on a synaptic architecture derived from a onedimensional arrangement [20]. Realistic networks take into account the two or three-dimensional structure of the physical space. In this section, we show that higher-dimensional networks also support the propagation of fast and slow pulses, and that the wave speed is determined by a generalized version of Eq. (5).

We consider a network on the $d$-dimensional square lattice $Z^{d}$. Neurons are located at the nodes of the lattice. A neuron $\mathbf{x}$ receives a connection from another neuron $\mathbf{y}$ if $\operatorname{dist}(\mathbf{x}, \mathbf{y}) \leqslant R$, where dist is a distance on $\mathbb{Z}^{d}$ (here we use the usual Euclidian distance) and $R>0$ determines the extent of the coupling. We define a plane wave solution as one for which

$$
v(\mathbf{x}, t)=V\left(t-\frac{\mathbf{n} \cdot \mathbf{x}}{c}\right),
$$

where $v(\mathbf{x}, t)$ is the time course of the membrane potential of the neuron located at $\mathbf{x}, \mathbf{n} \in \mathbb{R}^{d}$ is a unitary vector that gives the direction of propagation, $c$ is the wave speed, and the dot denotes the usual scalar product on $\mathbb{R}^{d}$. The expression of the wave profile is given by 

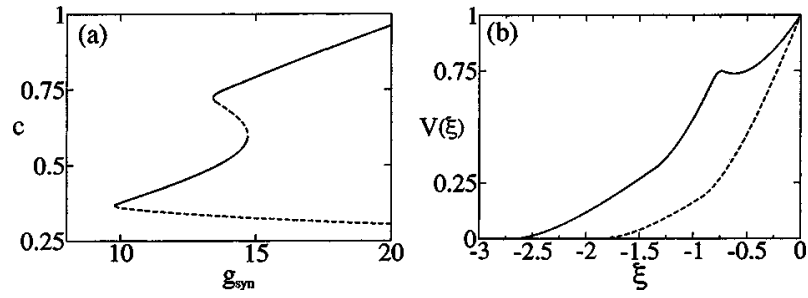

FIG. 7. Propagation of pulse waves in a two-dimensional network. We consider waves that propagate in the direction $\theta=45^{\circ}$, i.e., $\mathbf{n}=1 / \sqrt{2}(1,1)$. (a) Wave speed $c$ as a function of the coupling parameter $g_{s y n}$, obtained by solving (15) numerically. (b) Profile of the slow (solid line) and the fast (dashed line) plane waves as a function of the traveling wave coordinate $\xi$ for $\xi<0$, calculated using Eq. (14). Parameters are $\vartheta=1, \tau=0.5, \tau_{r}=1.9, \tau_{d}=0.1$ and (b) $g_{\text {syn }}=14$. The speed of the two stable waves is $c=0.521$ (slow wave) and 0.755 (fast wave). The synaptic connectivity is specified by the Euclidian distance $\operatorname{dist}(\mathbf{x}, \mathbf{y})=\left[\Sigma_{i}\left(x_{i}-y_{i}\right)^{2}\right]^{1 / 2}$ with the radius $R=2$. The synaptic weights are scaled according to $\omega(\mathbf{x}, \mathbf{y})$ $=\exp [-\operatorname{dist}(\mathbf{x}, \mathbf{y})]$.

$$
V(\xi)=\eta(\xi)+g_{\text {syn }} \sum_{\operatorname{dist}(\mathbf{x}, \mathbf{y}) \leqslant R} \omega(\mathbf{x}, \mathbf{y}) \epsilon\left(\xi+\frac{\mathbf{n} \cdot \mathbf{x}}{c}\right),
$$

where $\xi=t-\mathbf{n} \cdot \mathbf{x} / c$ is the traveling wave coordinate. The selfconsistency equation for $c$ is derived from the threshold condition, namely,

$$
\sum_{\operatorname{dist}(\mathbf{x}, \mathbf{y}) \leqslant R} \omega(\mathbf{x}, \mathbf{y}) \epsilon\left(\frac{\mathbf{n} \cdot \mathbf{x}}{c}\right)=\frac{\vartheta}{g_{s y n}},
$$

which can be seen as a generalization of Eq. (5). The wave speed depends on the direction of propagation as well as on the connectivity pattern. The dependence on the direction of propagation is not seen in continuous models where the neurons are packed in a dense manner, leading to an isotropic symmetry.

A numerical study of Eq. (15) reveals a behavior similar to the one-dimensional case. In particular, both fast and slow waves can propagate, and the number of slow wave solutions depends on the connectivity pattern and the direction of propagation. Figure 7 shows the time course of the voltage for a fast and a slow wave in a two-dimensional network. The predictions are in excellent agreement with a direct integration of Eq. (1) (not shown).

\section{CONCLUSION}

The discrete spatial connectivity is a natural property of neural networks that could lead to unexpected features. It is largely believed that purely excitatory networks do not allow for the propagation of slow traveling pulses. Surprisingly we found that considering a discrete spatial connectivity leads to the existence of a family of slow waves parametrized by the number of presynaptic units. These slow waves are characterized by a nonmonotonic increase of the voltage before the threshold is reached, due to a precise arrival of the successive EPSPs. The presence of weak noise does not alter our results, and moreover we show that a moderate amount of noise can induce both propagation failure or switching between different stable propagating modes. These new properties emerge when considering a limited number of presynaptic cells, relevant to the study of sparsely connected networks. In continuum models with spatially decaying connectivity, the propagation of slow waves is achieved with the aid of an inhibitory population, whereas excitatory networks can exhibit only fast waves.

Discrete neural networks have been introduced in the study of synfire chains [21,22]. Theoretical analyses of traveling waves in discrete networks with synaptic coupling are scarce [23]. Mathematical tools are well developed for the analysis of continuum media and the determination of traveling wave solutions is often related to a boundary value problem. However we have shown that an analytical treatment is also possible for discrete media. This paper provides a first step in the study of traveling pulses in discrete networks of simplified integrate-and-fire neurons. The theoretical framework can be easily generalized to include different aspects of neuronal dynamics.

\section{ACKNOWLEDGMENTS}

We are thankful to W. Gerstner for helpful discussions. This research was supported by Grant No. 2100-065269 from the SNF.

\section{APPENDIX A: VELOCITY OF THE FAST WAVE FOR NEAREST-NEIGHBOR COUPLING}

Using the analytical form of $\epsilon$,

$\epsilon(t)$

$$
\left\{\begin{array}{l}
0, \quad t<0 \\
\frac{\tau}{\tau_{r}}\left[t+\tau\left(e^{-t / \tau}-1\right)\right], \quad 0 \leqslant t \leqslant \tau_{r} \\
\tau\left(1+\frac{\tau_{r}+\tau-t}{\tau_{d}}\right)+\frac{\tau^{2}}{\tau_{r}} e^{-t / \tau} \\
-\left(\frac{\tau^{2}}{\tau_{r}}+\frac{\tau^{2}}{\tau_{d}}\right) e^{-\left(t-\tau_{r}\right) / \tau}, \quad \tau_{r} \leqslant t \leqslant \tau_{r}+\tau_{d} \\
{\left[\frac{\tau^{2}}{\tau_{r}}+\frac{\tau^{2}}{\tau_{d}} e^{\left(\tau_{r}+\tau_{d}\right) / \tau}-\left(\frac{\tau^{2}}{\tau_{r}}+\frac{\tau^{2}}{\tau_{d}}\right) e^{\tau_{r} / \tau}\right] e^{-t / \tau}, \quad \tau_{r}+\tau_{d} \leqslant t,}
\end{array}\right.
$$

expression (9) takes the form [we neglect the domain $c<\left(\tau_{r}+\tau_{d}\right)^{-1}$ since it is lower than the minimal wave speed, see below]

$$
\begin{gathered}
z+\gamma e^{-z}=a, \quad\left(\tau_{r}+\tau_{d}\right)^{-1} \leqslant c \leqslant \tau_{r}^{-1}, \\
z+e^{-z}=b, \quad \tau_{r}^{-1} \leqslant c,
\end{gathered}
$$

and the solution is given by

$$
c=\left\{\begin{array}{cc}
\frac{1}{\tau\left[W_{-1}\left(-\gamma e^{-a}\right)+a\right]}, & \left(\tau_{r}+\tau_{d}\right)^{-1} \leqslant c \leqslant \tau_{r}^{-1} \\
\frac{1}{\tau\left[W_{0}\left(-e^{-b}\right)+b\right]}, & \tau_{r}^{-1} \leqslant c,
\end{array}\right.
$$

where 


$$
\begin{gathered}
a=1+\left(\tau_{r}+\tau_{d}\right) / \tau-\left(\tau_{d} \vartheta\right) /\left(\tau^{2} g_{s y n}\right), \\
b=1+\left(\tau_{r} \vartheta\right) /\left(\tau^{2} g_{s y n}\right), \\
\gamma=\left(1+\tau_{d} / \tau_{r}\right) e^{\tau_{r} / \tau}-\tau_{d} / \tau_{r} .
\end{gathered}
$$

The critical coupling corresponds to the situation where $\max \{\epsilon(t)\}=\vartheta=1$, which yields

$$
g_{s y n}^{*}=\frac{1}{\epsilon\left(t^{*}\right)},
$$

where $t^{*}=\tau_{r}+\tau \ln \left[1+\left(\tau_{d} / \tau_{r}\right)\left(1-e^{-\tau_{r} / \tau}\right)\right]$ is the time to peak of $\epsilon$, from which we recover Eq. (11). Having checked that $t^{*}$ $\in\left(\tau_{r}, \tau_{r}+\tau_{d}\right)$, we can introduce this expression in Eq. (A1) to obtain the minimal wave speed

$$
c^{*}=\frac{1}{\tau\left[W_{-1}\left(-\gamma e^{-a^{*}}\right)+a^{*}\right]},
$$

where $a^{*}=1+t^{*} / \tau$. It is then easily checked that $\gamma e^{a^{*}}=e^{-1}$, so that $W_{-1}\left(-\gamma e^{-a^{*}}\right)=-1$, and we finally obtain the expected value $c^{*}=1 / t^{*}$.

\section{APPENDIX B: ASYMPTOTIC APPROXIMATION OF THE FAST WAVE SPEED}

For large values of $g_{s y n}$ one expects to obtain large speed values. Following Ermentrout [4], we expand $\epsilon$ in Taylor series about 0 in Eq. (5). If $p$ is the order of the first nonzero term in the expansion of $\epsilon(t)$ at $t=0$, we have

$$
\frac{1}{p ! c^{p}} \epsilon^{(p)}(0) \sum_{j=1}^{N} \omega_{j} j^{p}+O\left(\frac{1}{c^{p+1}}\right)=\frac{\vartheta}{g_{s y n}},
$$

where $\epsilon^{(p)}(0)$ is the derivative of order $p$ of $\epsilon(t)$ at $t=0$. Thus, we obtain the power law

$$
c^{p}=K g_{s y n}
$$

where $K$ is given by

$$
K=\frac{1}{p ! \vartheta} \epsilon^{(p)}(0) \sum_{j=1}^{N} \omega_{j} j^{p} .
$$

Using Eq. (8), $K$ can be expressed with the derivatives of $\alpha$ at $t=0$ and $q=p-1$ is the order of the first nonzero term in the expansion of $\alpha$.
[1] R. Mirollo and S. Strogatz, SIAM (Soc. Ind. Appl. Math.) J. Appl. Math. 50, 1645 (1990).

[2] L. Abbott and C. van Vreeswijk, Phys. Rev. E 48, 1483 (1993).

[3] N. Brunel, J. Comput. Neurosci. 8, 183 (2000).

[4] G. B. Ermentrout, J. Comput. Neurosci. 5, 191 (1998).

[5] P. C. Bressloff, Phys. Rev. Lett. 82, 2979 (1999).

[6] D. Golomb and G. B. Ermentrout, Proc. Natl. Acad. Sci. U.S.A. 96, 13480 (1999).

[7] P. C. Bressloff, J. Math. Biol. 40, 169 (2000).

[8] W. M. Kistler, Phys. Rev. E 62, 8834 (2000).

[9] D. Golomb and G. B. Ermentrout, Phys. Rev. Lett. 86, 4179 (2001).

[10] R. D. Chervin, P. Pierce, and B. W. Connors, J. Neurophysiol. 60, 1695 (1988).

[11] D. Golomb and Y. Amitai, J. Neurophysiol. 78, 1199 (1997).

[12] U. Kim, T. Bal, and D. A. McCormick, J. Neurophysiol. 74, 1301 (1995).

[13] D. Golomb, X. J. Wang, and J. Rinzel, J. Neurophysiol. 75, 750 (1996).

[14] A. Destexhe, T. Bal, and D. A. McCormick, J. Neurophysiol. 76, 2049 (1996).
[15] R. D. Traub, J. G. R. Jeffrey, and R. Miles, J. Neurophysiol. 472, 267 (1993).

[16] J. P. Keener, SIAM (Soc. Ind. Appl. Math.) J. Appl. Math. 47, 556 (1987).

[17] W. Gerstner and W. Kistler, Spiking Neurons Models: Single Neurons, Populations, Plasticity (Cambridge University Press, Cambridge, 2002).

[18] D. Golomb and G. B. Ermentrout, Phys. Rev. E 65, 061911 (2002).

[19] R. M. Corless, G. H. Gonnet, D. E. G. Hare, D. J. Jeffrey, and D. E. Knuth, Adv. Comput. Math. 5, 329 (1996).

[20] Discrete networks do not present a spatial organization in the sense that every network on $Z^{d}$ can be reduced to a onedimensional network (on $\mathbb{Z}$ ). However, this transformation leads to a meaningless synaptic connectivity of the "equivalent" one-dimensional network.

[21] M. Abeles, Corticonics (Cambridge University Press, Cambridge, 1991).

[22] M. Diesmann, M.-O. Gewaltig, and A. Aertsen, Nature (London) 402, 529 (1999).

[23] A. Tonnelier, J. Math. Biol. 44, 87 (2002). 